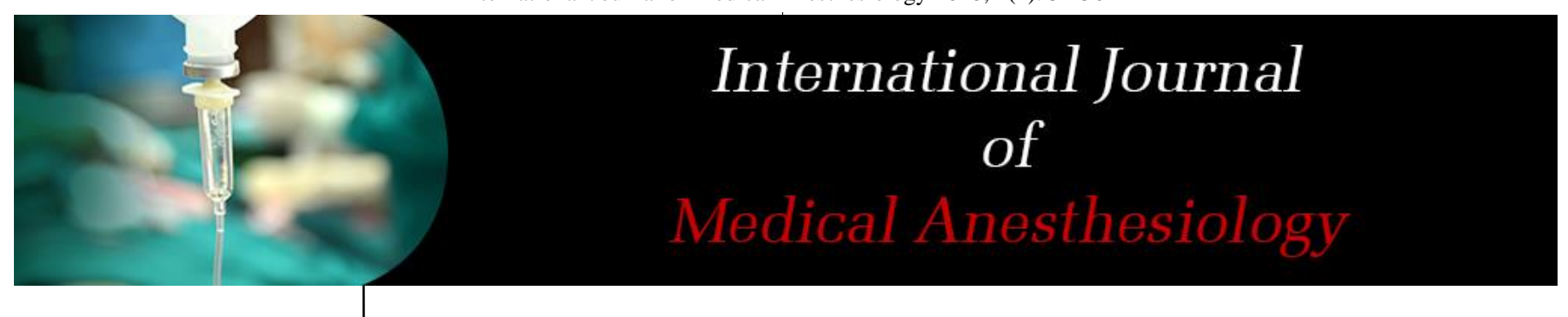

E-ISSN: 2664-3774

P-ISSN: 2664-3766

www.anesthesiologypaper.com

IJMA 2018; 1(2): 32-36

Received: 10-05-2018

Accepted: 16-06-2018

Dr. Pankaj Sharma

Junior Consultant,

Department of

Anaesthesiology, Metro

Hospital and Cancer

Research Center, Jabalpur,

Madhya Pradesh, India

Corresponding Author:

Dr. Pankaj Sharma

Junior Consultant,

Department of

Anaesthesiology, Metro

Hospital and Cancer

Research Center, Jabalpur,

Madhya Pradesh, India

\section{Double blind controlled clinical trial of comparison of levobupivacaine versus levobupivacaine with ketamine in elective caesarean section}

\section{Dr. Pankaj Sharma}

\section{DOI: https://doi.org/10.33545/26643766.2018.v1.i2a.214}

\begin{abstract}
Aim: The aim of the present study to comparison of levobupivacaine alone versus levobupivacaine with ketamine in subcutaneous infiltration for postoperative analgesia in lower segment caesarean section.

Material and Methods: A randomized double blind controlled study conducted in the Department of Anaesthesiology, Metro Hospital and Cancer Research Center, Jabalpur, Madhya Pradesh, India. A total of 100 adult parturient of Physical status II or III as per the American society of anesthesiologists (ASA) without any medical or obstetrical problems, and scheduled for cesarean section under spinal anesthesia were included in this study. Parturients were randomized to one of the two groups (50 each) according to computer generated random numbers kept in separate, sealed, and numbered envelopes. Group A parturients received subcutaneous surgical wound infiltration with a solution of $0.5 \%$ levobupivacaine at $2 \mathrm{mg} / \mathrm{kg}$ body weight (rounded to nearest multiple of 10) to a maximum of $150 \mathrm{mg}$ (maximum safe dose) diluted with normal saline to a total of $32 \mathrm{ml}$. Group B parturients received subcutaneous surgical wound infiltration with a solution of $0.5 \%$ levobupivacaine $2 \mathrm{mg} / \mathrm{kg}$ body weight (rounded to nearest multiple of 10) to a maximum of $150 \mathrm{mg}$ plus ketamine $1 \mathrm{mg} / \mathrm{kg}$ body weight diluted with normal saline to a total volume of $32 \mathrm{ml}$.

Results: We observed that both the groups were comparable with respect to demographic data. The zero hour (baseline) mean heart rates were comparable between groups $\mathrm{A}$ and $\mathrm{B}(P=0.957)$. The mean heart rate of group A was higher than that of group B which was statistically insignificant at majority of the time points except at $4^{\text {th }}$ and $6^{\text {th }}$ hour post-operative. The intra group comparison of mean heart rate showed a gradual decrease in values across time in both the groups but was more prominent in group B. Correspondingly, parturients in group A had higher mean VAS scores than those in group B at all-time intervals and statistically significant difference were observed at 1, 4, 6, and 12 hours.

Conclusion: We concluded that the ketamine is an effective adjunct modality to levobupivacaine for local wound infiltration in terms of superior pain relief, lesser need for rescue opioid analgesia, and no major side effects.
\end{abstract}

Keywords: Levobupivacaine, multimodal analgesia, N-Methyl-D-Aspartate antagonist, opioid consumption, subcutaneous wound infiltration

\section{Introduction}

Post-operative pain is inevitable after major upper abdominal surgeries like open cholecystectomy. Post-operative pain may cause stress response to body and respiratory or cardiac complications ${ }^{[1-3]}$. So, post-operative pain should be controlled as early as possible Post-operative analgesia is important part of optimal peri-operative management. Currently various methods are available for post-operative pain control like epidural analgesia, intravenous analgesia and patient controlled analgesia pump ${ }^{[4]}$. Opioids are mainstay of post-operative pain control but are associated with some adverse side effects like respiratory depression, sedation, nausea and vomiting ${ }^{[5-7]}$. Non-steroidal anti-inflammatory drugs are less effective as sole analgesic after upper abdominal surgeries. Local anesthetic methods are more useful than intravenous analgesia with less side effects irrespective of surgical procedure $^{[8]}$.

Now-a-days, wound infiltration with local anesthetic drugs is widely used in various surgeries as a part of optimal post-operative pain control ${ }^{[9,10]}$. Wound infiltration is safe, effective and inexpensive method of post-operative pain control. It provides immediate analgesia lasting for few hours without major side effects ${ }^{[11,12]}$. 
The levorotatory isomers were shown to have a safer pharmacological profile with less cardiac and neurotoxic adverse effects $[13,14]$. The decreased toxicity of levobupivacaine is attributed to its faster protein binding rate ${ }^{[15]}$. The pure $S(-)$ enantiomers of bupivacaine, i.e., ropivacaine and levobupivacaine were thus introduced into the clinical anesthesia practice. Levobupivacaine has been recently introduced into Indian market and is being widely used in various health set-ups. Such an increased usage mandates documentation of evidence based literature with regards to risk and safety concerns as well as clinical issues related to levobupivacaine. The aim of the present study to comparison of levobupivacaine alone versus levobupivacaine with ketamine in subcutaneous infiltration for postoperative analgesia in lower segment cesarean section.

\section{Material and Methods}

A randomized double blind controlled study conducted in the Department of Anaesthesiology, Metro Hospital and Cancer Research Center, Jabalpur, Madhya Pradesh, India, after taking the approval of the protocol review committee and institutional ethics committee.

\section{Methodology}

A total of 100 adult parturients of Physical status II or III as per the American society of anesthesiologists (ASA) without any medical or obstetrical problems, and scheduled for cesarean section under spinal anesthesia were included in this study. Uncooperative, unwilling parturients, and those with history of anaphylaxis to local anesthetics, opioids and/or drugs to be used, current or past history of drug abuse, psychiatric disease with body weight more than $100 \mathrm{Kg}$, and unable to understand the Visual Analog Scale (VAS) were excluded from the study.

Parturients were randomized to one of the two groups (50 each) according to computer generated random numbers kept in separate, sealed, and numbered envelopes. Group A parturients received subcutaneous surgical wound infiltration with a solution of $0.5 \%$ levobupivacaine at 2 $\mathrm{mg} / \mathrm{kg}$ body weight (rounded to nearest multiple of 10) to a maximum of $150 \mathrm{mg}$ (maximum safe dose) diluted with normal saline to a total of $32 \mathrm{ml}$. Group B parturients received subcutaneous surgical wound infiltration with a solution of $0.5 \%$ levobupivacaine $2 \mathrm{mg} / \mathrm{kg}$ body weight (rounded to nearest multiple of 10) to a maximum of 150 $\mathrm{mg}$ plus ketamine $1 \mathrm{mg} / \mathrm{kg}$ body weight diluted with normal saline to a total volume of $32 \mathrm{ml}$. An anesthetist not involved in the conduct of anesthesia and postoperative management prepared the study drugs and handed over to the surgeon for subcutaneous infiltration prior to skin closure under all aseptic precautions. A blinded observer assessed the postoperative pain relief up to 24 hours after the surgical procedure. A thorough preanesthetic check-up was conducted before the surgery which comprised of detailed history, general physical examination, and systemic examination. Routine investigations (complete hemogram, coagulation profile, random blood sugar) and other investigations if indicated were done prior to surgery. The VAS was shown and the scoring system was explained preoperatively. The parturients were informed before surgery that they can request for an analgesic after surgery if they feel pain and they can choose to withdraw from the study at any time.
After shifting the parturients to the operation theater in left lateral position, prior to subarachnoid block, pulse rate (P.R), non-invasive blood pressure (NIBP), respiratory rate (R.R), oxygen saturation $\left(\mathrm{SpO}_{2}\right)$, and electrocardiography (ECG) were recorded. These parameters were monitored throughout the procedure and recorded every 10 minutes. An intravenous access was achieved and preloading with 10 $\mathrm{ml} / \mathrm{kg}$ body weight with balanced salt solution was done. Thereafter, subarachnoid block was given under full aseptic precautions in sitting position. A 26 Gauge Quincke's needle was introduced into the subarachnoid space at L3-4/L4- 5 vertebral level. With the needle orifice cephalad and after confirmation of free flow of CSF, $2.0 \mathrm{ml}$ of $0.5 \%$ heavy bupivacaine was injected through the spinal needle, which was withdrawn after the injection was given and the parturient was then turned supine. Surgery was allowed to proceed after sensory block was achieved up to level of $\mathrm{T}_{4}$ and motor block to level of modified bromage scale of 3 . In case of partial/failed spinal anesthesia, general anesthesia was administered and the parturient was excluded from the study. At the end of surgery, parturients received subcutaneous skin infiltration of the study drug as per random group allocation in a blinded manner by the surgeon prior to skin closure.

Parturients were continuously monitored for heart rate, blood pressure, respiratory rate, and oxygen saturation. Postoperative pain scores and analgesic requirement were recorded along with hemodynamic parameters immediately after shifting to postoperative recovery room at $0 \mathrm{~min}, 30$ mins, 1, 2, 4, 6, 8, 12, 16, 20, and 24 hours, respectively. Postoperatively, all parturients received slow infusion in $100 \mathrm{ml}$ saline of diclofenac sodium $75 \mathrm{mg}$ at $0 \mathrm{~min}$ and, thereafter, 8 hourly as part of multimodal postoperative analgesia regimen. Any parturient with VAS greater than or equal to 4 or at any point of time complained of pain was administered with $50 \mathrm{mg}$ intravenous injection of tramadol, a rescue analgesic. Time of first rescue analgesic (FRA) request was noted. If the parturient still reported VAS $\geq 4$ after 1 hour of receiving tramadol, similar doses were repeated up to a maximum of $100 \mathrm{mg}$ in contiguous 4 hours or $400 \mathrm{mg}$ in 24 hours. The total rescue analgesic consumption for the 24 hours after surgery was recorded.

The observer (anesthesia resident posted in postanesthesia care unit) who recorded the postoperative vitals and analgesic consumption was blinded to the group allocation of the parturients to maintain the double blind nature of the study. The outcomes of the parturients were evaluated in terms of quality of pain relief (as assessed by VAS score) and time of FRA administration, number of times rescue analgesic given, and the total consumption volume of analgesic 24 hours postoperatively. Parturients were also evaluated for any adverse effects. The primary outcome, postoperative pain relief was measured using the VAS scale and the total analgesic consumption during the 24 hours postoperative period. The secondary outcome, that is, patient satisfaction score (PSS) was assessed postoperatively at 24 hours and was subjectively graded as:Excellent (4), Good (3), Moderate (2), Poor (1).

\section{Statistical analysis}

Data were described in terms of number and percentages. The mean and standard deviation were computed. Comparison of quantitative variables was done using Student $t$-test. For comparing categorical data, Chi-square or 
exact test was performed as applicable. A probability value ( $P$ value) of less than 0.05 was considered statistically significant. SPSS version 20.0 was used for all statistical calculations.

\section{Results}

We observed that both the groups were comparable with respect to demographic data. The zero hour (baseline) mean heart rates were comparable between groups $\mathrm{A}$ and $\mathrm{B}(P=$ 0.957). The mean heart rate of group A was higher than that of group B which was statistically insignificant at majority of the time points except at $4^{\text {th }}$ and $6^{\text {th }}$ hour post-operative. The intra group comparison of mean heart rate showed a gradual decrease in values across time in both the groups but was more prominent in group B [Table 1]. Correspondingly, parturients in group A had higher mean VAS scores than those in group B at all-time intervals and statistically significant difference were observed at 1, 4, 6, and 12 hours [Table 2]. The mean time to FRA of group A was at $3.35 \pm 2.21$ hours (194 mins) while that of group B was at $4.97 \pm 2.36$ hours (286 mins). This difference was statistically significant $(\mathrm{P}=0.043)$. Thus, parturients in Group B complained of pain 1.6 hours later than the parturients in group A [Table 3]. In group B with ketamine as an adjuvant to levobupivacaine, only $45 \%$ of the parturients demanded rescue analgesia, whereas nearly $95 \%$ of the parturients needed rescue analgesia in group A which received levobupivacaine alone. [Table 4] Parturients in group A consumed a mean total opioid dose of $97.63 \pm 38.26$ $\mathrm{mg}$ in 24 hours compared to $62.12 \pm 23.67 \mathrm{mg}$ in group B. Thus, statistically significant higher opioid consumption was observed in group A than in group B $(P=0.002$, Table 5). There were $7 \%$ parturient in Group A compared to $24 \%$ in Group B in whom the PSS was of excellent quality and $25 \%$ in Group A and 45\% in Group B graded the PSS with good quality. Thus, the difference in patient satisfaction score was statistically significant between the two groups ( $P$ $=0.007$, table 6 ).

Table 1: Trends in post-operative mean heart rate

\begin{tabular}{|c|c|c|c|c|c|}
\hline HR & \multicolumn{2}{|c|}{ Group A } & \multicolumn{2}{c|}{ Group B } & P \\
\hline & Mean & SD & Mean & SD & \\
\hline $0 \mathrm{~h}$ & 90.83 & 14.22 & 91.21 & 11.81 & 0.957 \\
\hline $30 \mathrm{~min}$ & 91.15 & 14.11 & 90.12 & 12.68 & 0.752 \\
\hline $1 \mathrm{~h}$ & 92.49 & 16.39 & 89.83 & 13.51 & 0.478 \\
\hline $2 \mathrm{~h}$ & 94.55 & 15.97 & 87.80 & 11.85 & 0.069 \\
\hline $4 \mathrm{~h}$ & 94.95 & 15.96 & 86.83 & 11.21 & 0.032 \\
\hline $6 \mathrm{~h}$ & 93.42 & 13.75 & 86.60 & 9.57 & 0.029 \\
\hline $8 \mathrm{~h}$ & 86.75 & 12.87 & 87.83 & 9.72 & 0.752 \\
\hline $12 \mathrm{~h}$ & 84.82 & 11.06 & 85.07 & 9.92 & 0.963 \\
\hline $16 \mathrm{~h}$ & 83.42 & 11.63 & 83.42 & 7.87 & 0.934 \\
\hline $20 \mathrm{~h}$ & 83.96 & 11.75 & 83.72 & 8.32 & 0.922 \\
\hline $24 \mathrm{~h}$ & 84.12 & 9.87 & 82.81 & 8.84 & 0.569 \\
\hline
\end{tabular}

Table 2: Mean VAS scores at various time intervals

\begin{tabular}{|c|c|c|c|c|c|}
\hline \multirow{2}{*}{ VAS } & \multicolumn{2}{|c|}{ Group A } & \multicolumn{2}{c|}{ Group B } & P value \\
\cline { 2 - 6 } & Mean & SD & Mean & SD & \\
\hline $0 \mathrm{~h}$ & 1.35 & 1.61 & 0.71 & 0.99 & 0.129 \\
\hline $30 \mathrm{~min}$ & 1.06 & 1.22 & 0.62 & 0.87 & 0.127 \\
\hline $1 \mathrm{~h}$ & 1.79 & 1.62 & 0.63 & 1.19 & 0.005 \\
\hline $2 \mathrm{~h}$ & 2.05 & 1.87 & 1.11 & 1.37 & 0.081 \\
\hline $4 \mathrm{~h}$ & 2.41 & 2.13 & 1.05 & 1.63 & 0.008 \\
\hline $6 \mathrm{~h}$ & 1.89 & 1.70 & 0.84 & 1.62 & 0.006 \\
\hline $8 \mathrm{~h}$ & 2.11 & 2.21 & 1.27 & 1.93 & 0.081 \\
\hline $12 \mathrm{~h}$ & 1.25 & 1.69 & 0.16 & 0.53 & 0.001 \\
\hline $16 \mathrm{~h}$ & 0.86 & 1.73 & 0.15 & 0.53 & 0.058 \\
\hline $20 \mathrm{~h}$ & 0.67 & 1.42 & 0.15 & 0.53 & 0.112 \\
\hline $24 \mathrm{~h}$ & 0.21 & 0.71 & 0.15 & 0.53 & 0.390 \\
\hline
\end{tabular}

VAS - Visual analogue scale, $P<0.05$ significant

Table 3: Mean time to first rescue analgesia (FRA) and VAS at FRA

\begin{tabular}{|c|c|c|c|c|c|}
\hline & \multicolumn{2}{|c|}{ Group A } & \multicolumn{2}{c|}{ Group B } & P value \\
\hline & Mean & SD & Mean & SD & \\
\hline $\begin{array}{c}\text { Time to first rescue } \\
\text { analgesic consumption (h) }\end{array}$ & 3.35 & 2.21 & 4.97 & 2.36 & 0.043 \\
\hline VAS AT FRA & 4.85 & 1.26 & 4.67 & 0.59 & 0.597 \\
\hline
\end{tabular}

Table 4: Total rescue analgesic consumption in $24 \mathrm{~h}(\mathrm{mg})$

\begin{tabular}{|c|c|c|c|c|c|}
\hline & \multicolumn{2}{|c|}{ Group A } & \multicolumn{2}{|c|}{ Group B } & P value \\
\hline & Mean & SD & Mean & SD & \\
\hline $\begin{array}{c}\text { Total opioid analgesic } \\
\text { consumption in 24 h (mg) }\end{array}$ & 97.63 & 38.26 & 62.12 & 23.67 & 0.002 \\
\hline
\end{tabular}

Table 5: Percentage of parturient requiring rescue analgesic in each group

\begin{tabular}{|c|c|c|}
\hline & No & Yes \\
\hline Group A & 5 & 95 \\
\hline Group B & 55 & 45 \\
\hline
\end{tabular}

Table 6: Percentage of parturient with patient satisfaction score (PSS)

\begin{tabular}{|c|c|c|c|c|}
\hline & Score 1 & Score 2 & Score 3 & Score 4 \\
\hline Group A & 10 & 58 & 25 & 7 \\
\hline Group B & 0 & 31 & 45 & 24 \\
\hline
\end{tabular}

\section{Discussion}

Subcutaneous wound infiltration with local anesthetics is effective, safe, inexpensive, and without the need for expertise. A systemic review and meta- analysis substantiated the analgesic efficacy of various local anesthetic wound infiltration techniques for postoperative analgesia following cesarean section. They observed a statistically significant reduction in postoperative pain scores and total opioid consumption in 24 hours with local anesthetic wound infiltration ${ }^{[16]}$. We used levobupivacaine alone and levobupivacaine plus ketamine for local wound infiltration after cesarean section in view of their analgesic and anti-inflammatory properties along with a lesser cardiotoxic profile. 
In our study on 100 parturients, none were excluded and both the groups were statistically comparable with respect to age, weight, and ASA grade. We observed that patients in group $B(L+K)$ experienced a postoperatively pain- free period of up to 286 mins whereas patients in group A (L) demanded rescue analgesia at 194 mins. This portrays that local wound infiltration provides adequate analgesia and addition of ketamine to levobupivacaine significantly contributes in the prolongation of pain- free period $(P=$ 0.043). Though we observed statistically significant prolonged times to FRA, the mean VAS score at FRA in both the groups was comparable- $(4.85 \pm 1.26)$ in group A and $4.67 \pm 0.59$ in group B). This implies that while levobupivacaine conferred profound analgesic effect in both the groups, addition of ketamine helped in prolonging the time required for FRA. Abdallah et al. evaluated the analgesic efficacy of preincisional infiltration with ketamine or levobupivacaine in 48 patients undergoing abdominal hysterectomy. They observed an increased duration of analgesia for up to 158 mins with ketamine and 127 mins with levobupivacaine $(P=0.001)$. The time to FRA in their study group using levobupivacaine was shorter compared to our study group A which can be attributed to the use of less volume and concentration of levobupivacaine solution for infiltration, that is, $20 \mathrm{ml}$ and only $0.25 \%$ levobupivacaine in their study ${ }^{[17]}$.

The overall VAS scores of our study were higher in group A with statistically significant higher values at 1, 4, 6, and 12 hours, which correlated with a significant increase in heart rate at 4 and 6 hours in the same group. This translates that the group infiltrated with levobupivacaine alone had comparatively initial peaks of higher pain scores reflected by higher heart rates at the same time of observations, whereas the group which received ketamine as an adjunct to levobupivacaine for infiltration encountered less pain during the same period. Thus, addition of ketamine as an adjunct to levobupivacaine enhances its efficacy in terms of profound long- lasting postoperative analgesia.

We observed that only $45 \%$ of the participants demanded rescue analgesia in group B whereas $95 \%$ needed additional tramadol supplementation in group A. Corresponding to this, there was a statistically significant decrease in mean rescue analgesic consumption of tramadol in group B amounting to $63 \mathrm{mg}$, whereas it was $96 \mathrm{mg}$ in group A $(P=$ 0.002). These findings substantiate the opioid sparing effect of ketamine when used as an adjunct to levobupivacaine and an abatement in opioid- related side effects such as nausea, vomiting, pruritus, and sedation. Demiraran et al. studied 90 patients undergoing cesarean section under general anesthesia, where the wound was infiltrated with $20 \mathrm{ml}$ of $0.25 \%$ levobupivacaine at the end of surgery. They observed a total tramadol consumption of $483 \mathrm{mg}$ in the study group and $560 \mathrm{mg}$ in the placebo group $(P=0.07)$. The overall higher tramadol consumption in the study group than in group A can be attributed to the use of less concentration and lesser volume of levobupivacaine in their study. In addition, we used multimodal analgesia by giving intravenous injection of diclofenac $75 \mathrm{mg} 8$ hourly to all the parturients which was not a part of their study ${ }^{[18]}$.

A Cochrane review encompassing 20 studies on parturients who received wound infiltration following cesarean section under regional anesthesia observed a statistically significant decrease in morphine consumption at 24 hours compared to placebo. However, this analysis revealed no additional advantage in terms of patient satisfaction score upon addition of ketamine to continuous wound infiltration with $0.125 \%$ bupivacaine, where the catheter was placed above the fascia affecting the spread of drug ${ }^{[19]}$. On the contrary, we observed a statistically and clinically significant improvement in the patient satisfaction scores of group B compared to group A $(P=0.02)$ which can be credited to the use of higher concentration, that is, $0.5 \%$ of levobupivacaine with ketamine.

\section{Limitations}

The infiltration of local anesthetic was conducted by different surgeons, thus causing minor differences in the infiltration technique.

The duration of surgery that reflects the degree of tissue handling and inflammatory response which directly impacts on the degree of postoperative pain was not taken into account.

VAS score at movement was not recorded which could improve the analysis of pain and also the determine rate of early mobilization.

\section{Conclusion}

We concluded that the ketamine is an effective adjunct modality to levobupivacaine for local wound infiltration in terms of superior pain relief, lesser need for rescue opioid analgesia, and no major side effects.

\section{Reference}

1. Wightman JA. A prospective survey of the incidences of post-operative pulmonary complications. Br J Surg 1968;55:85-91.

2. Latimer RG, Dickman M, Day WC et al. Ventilatory patterns and pulmonary complications after upper abdominal surgery determined by pre-operative and post-operative computerized spirometry and blood gas analysis. Am J Surg 1971;122:622-32.

3. Sun JX, Bai KY, Liu YF et al. Effects of local wound infiltration with ropivacaine on post-operative pain relief and stress response reduction after open hepatectomy. World J Gastroenterol 2017;23(36):6733740.

4. Zhu $\mathrm{H}$, Wang $\mathrm{C}, \mathrm{Xu} \mathrm{C}$ et al. Influence of patientcontrolled epidural analgesia versus patient- controlled intravenous analgesia on post-operative pain control and recovery after gastrectomy for gastric cancer: A prospective randomized trial. Gastric Cancer 2013;16:193-200.

5. Bhardwaj S, Devgan S, Sood D et al. Comparison of local wound infiltration with ropivacaine alone or ropivacaine plus dexmedetomidine for post- operative pain relief after lower segment cesarean section. Anesth Essays Res 2017;11(4):940-45.

6. Dahl JB, Jeppesen IS, Jorgensen $\mathrm{H}$ et al. Intraoperative and post-operative analgesic efficacy and adverse effects of intrathecal opioids in patients undergoing cesarean section with spinal anesthesia: A qualitative and quantitative systematic review of randomized controlled trial. Anesthesiology 1999;91:1919-927.

7. Gehlin M, Tryba M. Risks and side effects of intrathecal morphine combined with spinal anesthesia: A meta-analysis. Anesthesia 2009;64:643-51.

8. Wu CL, Cohen SR, Richman JM et al. Efficacy of post- 
operative patient-controlled and continuous infusion epidural analgesia versus intravenous patient-controlled analgesia with opioids: A meta- analysis. Anesthesiology 2005;103:1079-088.

9. Scott NB. Wound infiltration for surgery. Anesthesia 2010;65S:67-75.

10. Lee KC, Lu CC, Lin SE et al. Infiltration of local anesthesia at wound site after single- incision laproscopic colectomy reduces post-operative pain and analgesic usage. Hepato Gastroenterology 2015;62:811-16.

11. Moiniche S, Mikkelsen S, Wetterslev J et al. A systematic review of incisional local anesthesia for post-operative pain after abdominal operations. Brit Anesth 1998;81:377-83.

12. Vigneau A, Salengro A, Berger J et al. A double blind randomized trial of wound infiltration with ropivacaine after breast cancer surgery with axillary nodes dissection. BMC Anesthesiol 2001;24:11-23.

13. Huang YF, Pryor ME, Mather LE, Veering BT. Cardiovascular and central nervous system effects of intravenous levobupivacaine and bupivacaine in sheep. Anesth Analg 1998;86:797-804.

14. Morrison SG, Dominguez JJ, Frascarolo P, Reiz S. A comparison of the electrocardiographic cardiotoxic effects of racemic bupivacaine, levobupivacaine, and ropivacaine in anesthetized swine. Anesth Analg 2000;90:1308-14.

15. Burm AG, Van Der Meer AD, Van Kleef JW, Zeijlmans PW, Groen K. Pharmacokinetics of the enantiomers of bupivacaine following intravenous administration of the racemate. Br J Clin Pharmacol 1994;38:125-9.

16. Adesope O, Ituk U, Habib AS. Local anesthetic wound in filtration for post-cesarean section analgesia: A systematic review and meta- analysis. Eur J Anaesthesiol 2016;33:731- 42.

17. Abdallah NM, Salama AK, Ellithy AM. Effects of preincisional analgesia with surgical site infiltration of ketamine or levobupivacaine in patients undergoing abdominal hysterectomy under general anesthesia; A randomized double blind study. Saudi J Anaesth 2017;11:267- 72 .

18. Demiraran Y, Albayrak M, Yorulmaz IS, Ozdemir I. Tramadol and levobupivacaine wound infiltration at cesarean delivery for postoperative analgesia. J Anesth 2013;27:175- 9 .

19. Bamigboye AA, Hofmeyr GJ. Local anesthetic wound in filtration and abdominal nerves block during cesarean section for postoperative pain relief. Cochrane Database Syst Rev 2009;3:CD006954. 